Relations industrielles

Industrial Relations

\title{
La grève de l'amiante. En collaboration, sous la direction de Pierre Elliott Trudeau, Les Editions Cité libre, 1956, 430 pp.
}

\section{Raymond Guérin}

Volume 11, numéro 4, septembre 1956

URI : https://id.erudit.org/iderudit/1022616ar

DOI : https://doi.org/10.7202/1022616ar

Aller au sommaire du numéro

Éditeur(s)

Département des relations industrielles de l’Université Laval

ISSN

0034-379X (imprimé)

1703-8138 (numérique)

Découvrir la revue

Citer ce compte rendu

Guérin, R. (1956). Compte rendu de [La grève de l'amiante. En collaboration, sous la direction de Pierre Elliott Trudeau, Les Editions Cité libre, 1956, 430 pp.] Relations industrielles / Industrial Relations, 11(4), 306-309.

https://doi.org/10.7202/1022616ar

Tous droits réservés @ C Département des relations industrielles de l’Université Laval, 1956
Ce document est protégé par la loi sur le droit d'auteur. L'utilisation des services d'Érudit (y compris la reproduction) est assujettie à sa politique d'utilisation que vous pouvez consulter en ligne.

https://apropos.erudit.org/fr/usagers/politique-dutilisation/ 
according to the end of social economy, every productive member is a subject and not an object of economic life."

"There are other aspects", the prelate stated, "that the Christian economist cannot ignore... First, there is the question of the displacement of the worker... Second, there is the new future that lies open to professionnal and technical teaching. Even here grave human and religious needs must be protected, for it is not without reason that the youth of the future, formed in a "technical spirit", is a matter of worry for those who have at heart the moral health of the society of tomorrow."

In concluding his letter, Msgr. Dell'Acqua called on Catholics to keep in mind the general good of the nation by opposing outdated methods, particular and selfish interests and by opposing with equal force "a blind expansion whose only drive is a thirst for profit".

\section{LIVRES ET REVUES}

La grève de l'amiante. En collaboration, sous la direction de Pierre Elliott Trudeau, Les Editions Cité libre, 1956, 430 pp.

Aussi paradoxale que la chose puisse paraître, ce n'est pas la grève de l'amiante qui constitue, à notre avis, le point tournant de l'histoire sociale de notre province, mais la parution d'un livre comme cette étude sur la grève de l'amiante que nous sommes appelé à recenser ici.

Parce que la publication d'une étude sociologique de cette envergure, avec cette acuité de vues et indépendance d'idées, aurait été impossible, il y a vingt ans, et même quinze, faute de chercheurs préparés en matière sociale et capables surtout de dire en toute franchise des faits qui heurtent à coup sûr le conformisme de front.

Parce qu'aussi, la grève de l'amiante - et les évènements subséquents et récents le confirment - demeurera, plutôt, croyons-nous. comme un point de référence dans l'éveil de la conscience sociale chez nous, ou encore une minute d'inattention, une limite extrême dépassée un moment, dans la politique de «containment» de la promotion ouvrière.

Mais voyons plutôt, chapître par chapître, cette puissante étude.

Dans la préface, M. Jean-Charles Falardeau fait une excellente présentation de l'ouvrage et des hommes qui lont mis au point. Des difficultés ren- contrées, des résultats obtenus. Il y élabore, cependant, une théorie des forces sociales dans cette Province qui relève plus de la géométrie que de la sociologie. Dire, par exemple, que «jusque là, dans le Québec, tout différend ouvrier, d'une certaine envergure auquel le syndicalisme confessionnel se trouvait mêlé était, à son point d'origine, défini, et à son point d'arrivée, réglé (officiellement ou officieusement) par la trinité patronat-gouvernement-Eglise ou par l'une ou l'autre des combinaisons deux par deux des trois partenaires: patronat-gouvernement, patronat-Eglise, gouvernement-Eghise » est peut-être fondé, mais demeure tout de même une affirmation gratuite que des études sociologiques vaudraient d'étayer. M. Falardeau est un homme de grand talent qui serait tout désigné nour de telles études.

L'étude de M. Pierre Elliott Trudeau qui suit, est la plus considérable du livre et peut-être la plus lucide et la plus franche à la fois qui ait été faite par l'un des nôtres sur les problèmes de l'industrialisation chez nous, sur les attitudes traditionnelles adoptées à ce sujet et sur les conséquences de ces attitudes dans l'évolution de notre penser et agir sociaux.

Cette étude mériterait d'être plus poussée, plus nuancée, certes, mais nous croyons qu'elle demeurerait fondamentalement vraie et juste. Elle tient en ceci: Notre Province a marqué une transformation profonde au cours des cinquante dernières années. D'agricole qu'elle était, elle est devenu industrielle. Or, pendant ce temps nos dirigeants et nos institutions firent comme si cette réalité sociologique ne se posait pas. Devant les problèmes que suscitait cette 
transformation dans notre société, l'attitude traditionnelle fut double: rejeter a priori toute solution qui apparaissait d'inspiration étrangère et rechercher la clé aux problèmes nouveaux dans un retour vers le passé.

Et M. Trudeau de faire parler les hommes et les institutions qui ont forgé cette double attitude concrétisée par cette déclaration d'il y a cinquante ans de Mgr Paquet: «Pendant que nos riyaux revendiquent... l'hégémonie de l'industrie et de la finance, nous ambitionnerons avant tout l'honneur de la doctrine et les palmes de l'apostolat », attitude qui instituait cette espèce de nationalisme spiritualiste qui allait fermer les voies à toute initiative dans la gestion de nos affaires économiques, et qui nous amenait par voie de conséquence à repousser tout système politique, toute pensée sociale qui ne serait pas venu de nous.

Et pendant ce temps, dans le domaine des solutions au problème global de l'industrialisation, on mettait et remettait de l'avant des formules comme le retour à la terre, l'entreprise familiale, le corporatisme, etc., formules de facilité ou d'irréalité comme l'avenir allait le démontrer.

Or, quand on songe que les nombreuses citations confirmant cette attitude de nos dirigeants et de nos institutions datent pour un grand nombre de quinze ans à peine, on ne peut manquer d'être édifié sur l'irréalisme de notre pensée sociale, économique ou nationale passée.

Mais pendant que nous repoussions d'en haut tout ce qui nous est étranger, notre jeunesse s'imprégnait de plus en plus d'américanisme dans tout ce qu'il a de moins bon; pendant que nous pratiquions le détachement des biens de ce monde, nous voyions nos richesses passer à l'étranger; pendant que nous nous refugions dans les constructions de la pensée pure, nous négligions les constructions tout court qui abriteraient nos familles.

Bref nous avons agi comme si notre peuple habitait quelqu'île éloignée du Pacifique dans une société d’hommes bons de nature à la Jean-Jacques Rousseau.

Reste, cependant, que les dividendes politiques d'une telle attitude: agriculturisme, anti-communisme... demeurent encore aujourd'hui fort plantureux.

Le mérite de M. Trudeau aura été d'avoir dit franchement notre faux acheminement passé afin que nous nous orientions mieux désormais dans la réa- lité sociale présente. Nous ne pouvons lui tenir rigueur du ton agressif qu'il emploie parfois et des affirmations qui exigeraient d'être plus nuancées.

M. Jean Gérin-Lajoie, en faisant l'histoire financière de la grève de l'amiante a su mettre en lumière, avec les chiffres disponibles, le double problème de l'écoulement de l'amiante sur le marché mondial et de la concurrence féroce que se livrèrent les producteurs en fin du siècle dernier et au début de ce siècle et des répercussions de cet état de choses sur les conditions ouvrières de travail. C'est ici que se vérifie plus que jamais cet expression de Pie XII lorsqu'il compare l'ouvrier à «la bille dans la main d'un joueur ». Une telle situation explique à coup sur la solidarité des ouvriers au moment de la grève quand l'on songe surtout que pour tout ouvrier, de quarante ans et plus, l'insécurité et l'insalubrité au travail avaient été le lot de toute sa vie antérieure.

Les chapîtres suivants sur l'histoire du syndicalisme dans l'industrie de l'amiante et de la grève elle-même par MM. Fernand Dumont et Gilles Beausoleil sont assurément les plus passionnants de tout le livre.

Commencé à la fin d'une soirée, je me souviens en avoir poursuivi la lecture d'un seul trait tout le long des premières heures de la nuit. Je me reportais pour l'intérêt que j'y prenais aux jours de ma première jeunesse alors que je dévorais les grands romans de chevalerie et d'aventures du temps, genre «Mouron rouge», «Sous la Terreur», etc.

Que de tâtonnements inutiles, que d'illusions bercées, que d'efforts incohérents, cette histoire rappelle tout au long, mais aussi quelle fusion d'âmes, quelle cohésion des esprits elle forge à travers toutes ses vicissitudes. L'histoire du syndicalisme de l'amiante est plus qu'une histoire, elle relève de l'épopée. Aussi, faut-il espérer que d'aussi admirables monographies s'écrivent sur les autres syndicalismes professionnels cheznous!

Quant à la grève même de l'amiante, bien que nous l'ayions suivie de loin, il est vrai, dans son évolution de chaque jour, elle nous fut quand même une révélation par l'abondance des détails qui nous avaient échappés, alors, et dont nous n'avions pas saisi toute la portée. Nous ne regrettons qu'une chose, c'est que les références aux textes cités ne soient pas indiquées à chaque fois. Nous croyons, à l'encontre de l'auteur, 
que ceci aurait contribué à donner un accent de véracité plus puissant encore à cette étude.

Le chapître $\mathrm{V}$, écrit en collaboration retrace l'histoire des négociations d'avant, de pendant et d'après la grève. La nature de ces négociations a contribué, il semble bien, à vérifier la méfiance des cuvriers à l'endroit des procédures d'arbitrage et qui fut assurément l'une des causes principales de la grève. Il appert, au récit, que les tractations arbitrales d'après grève furent avant tout de permettre aux perdants de sauver la face. Elles avaient duré en tout quatorze mois, mais souligné surtout l'importance nouvelle des relations de travail dans notre société industrialisée.

N'eut été l'intervenion de l'Eglise, on peut dire avec certitude que la grève de lamiante se serait terminée par un échec retentissant pour le syndicat ouvrier. C'est ce qui ressort de l'étude de $\mathbf{M}$. l'abbé Gérard Dion et qui constitue, dans cette perspective, l'une des plus importantes de tout le livre.

Certaines allusions, dans le texte, semblent indiquer que l'auteur n'a pas été étranger, à certains moments, à cette intervention. C'est le mérite de $\mathbf{M}$. l'abbé Dion d'avoir situé cette intervention dans ses motifs véritables et ses buts profonds.

$\mathrm{Au}$ grand motif de charité, «bien d'autres motifs " comme l'écrira Son Excellence Mgr Douville (p. 257) ont suscité l'intervention de l'Eglise dans le conflit de l'amiante. Deux motifs, à la lecture, se détachent de cette étude: a) \& le sort même de la CTCC (un organisme fondé et appuyé par l'Eglise) était lié à son issue 》 (pp. 245 et 253) et $b)$ le « jugement sur l'orientation doctrinale de la CTCC et l'attitude des prêtres » (p. 247) par le président de la Canadian Johns-Manville Co., un protestant et par certains laïcs (p. 254), et qui relevait, il va de soi, d'autorités plus compétentes.

Mais cette prise de position catégorique de l'Eglise qui sortait « de ce conflit grandit par sa sympathie envers les faibles », n'allait pas sans susciter de délicats problèmes dans ses rapports avec l'Etat dont la prise de position avait été non moins catégorique. Aussi. M. l'abbé Dion, qui est l'un de nos meilleurs sociologues, s'est-il gardé, comme tel, de tirer des conclusions qui n'auraient été que des conjectures. Il a laissé à l'histoire d'écrire les solutions ou les aggravations de ces problèmes.
La grève de l'amiante fut une grève illégale, mais il apparaît, au chapître suivant de M. Charles Lussier, que cette illégalité fut l'effet, aussi bien que l'occasion de multiples illégalités de la part de toutes les parties en cause, aussi bien Compagnie, Comission des Relations ouvrières que Gouvernement. $\mathrm{Ce}$ qui vérifie que l'on peut établir des relations de travail à la bonne franquette et qu'elles peuvent être excellentes si les parties sont en confiance. Mais si on veut absolument fonder ces relations sur la légalité, la fantaisie n'est plus possible désormais. Il faut qu'une porte soit ouverte ou fermée.

$\mathrm{Au}$ chapître de la grève et de la presse, par M. Gérard Pelletier, je songeai tout de suite à cette remarque de Sauvy: «Le coût très élevé de l'information a toujours favorisé les défenseurs du capital. La concentration des moyens de production a encore accentué ce désiquilibre $\gg .{ }^{1}$

La lenteur à se saisir de tout le problème, la fantaisie dans l'interprétation des évènements, la partialité voulue, dans certains cas, mais le plus souvent ignorante, dans la relation des faits, le silence surtout autour des causes et des buts de la grève semblent avoir été le fait de la presse en général dans cette affaire. Mais ce fut aussi. selon l'auteur, l'occasion d'un éveil de l'opinion publique au point que par la suite la presse fit preuve dans des conflits subséquents d'une plus grande objectivité. Cette constatation que l'auteur fait d'une plus grande maturité dans l'opinion publique est assurément consolante. Puisse-t-il dire vrai? Reste certain, comme le dit M. Pelletier que «si la presse jouait plus honnêtement, plus librement son rôle en notre pays, les conflits sociaux connaîtraient sans doute des solutions plus rapides et plus justes ».

«Six ans après», par M. Maurice Sauvé et «La Grève et le mouvement ouvrier » par M. Réginald Boisvert ont ce rapport entre eux que les résultats indiscutables obtenus par les ouvriers de l'amiante et rapportés par M. Sauvé, ont été dus à la solidarité du mouvement ouvrier comme les échecs subséquents ont été causés par la division des forces syndicales. La grève de l'amiante a constitué le point culminant d'une courbe que l'ont pourrait tracer de la soli-

\footnotetext{
(1) A. Sauvy, L'Opinion Publique, Coll. «Que Sais-Je?» 1956, p. 116.
} 
darité syndicale chez nous et dont la récente fusion au plan national ne cache tout de même pas la singularité de ce mariage de raison, mais qui pose, par ailleurs pour la CTCC un dilemne angoissant.

Ce qui fait constater à M. Boisvert: «Les germes de vie que la grève de l'amiante a semés, ou dont elle a révélé la présence chez nous n'ont pas encore produit la moisson qu'on en attendait ». Mais l'auteur est un «croyant » et ne cache pas, par ailleurs, son optimisme pour l'avenir (p. 257). Mais la sociologie est affaire du présent, et le présent est dans l'attente.

Quelles leçons tirer de cette grève de l'amiante? De quoi demain sera-t-il fait pour les travailleurs, pour notre société, notre nationalité? La grève de l'amiante a sûrement obligé à repenser bien des principes, à en tirer des conclusions jusque là impensées. L'avenir s'en éclaire d'un jour nouveau, mais cet avenir, déclare en épilogue M. Trudeau «s'imposera à nous avec les normes qu'il véhicule, ou bien nous nous taillerons une place dans cet avenir avec celles qui nous importent ». Ceux qui sont en place vous diront que la chose ne se pose pas, mais nous croyons qu'elle demeure douloureusement vraie pour ceux qui veulent honnêtement regarder autour d'eux.

Le volume est complété par des documents ayant traits à la grève de l'amiante, dont le fameux rapport Custos, oeuvre anonyme à l'adresse de Rome. et qui constitue l'une de ces pièces dont Machiavel, s'il revenait parmi nous, en aurait sûrement réclamé la paternité. Consolations: de tels rapports sont toujcurs à l'appendice des grands faits de l'histoire où des hommes confirment par leur petitesse la grandeur des évènements.

Cette oeuvre sur la grève de l'amiante est sûrement, malgré les réserves que nous avons mentionnées, une des pièces maîtresses de l'histoire sociale chez nous. Il ressort à sa lecture que les auteurs ont été amenés par une pensée, un désir et un élan commun à éclairer le débat pour d'autre chose que d'avoir raison. Des résultats obtenus sont dus à la valeur des auteurs, à leur esprit critique, malgré d'inévitables redites dans une oeuvre de ce genre.

La parution de ce volume, avons-nous dit au début, nous semble un évènement plus important que la grève elle-même. Elle marque une révolution dans le domaine de la recherche sociale et de l'étude approfondie des phénomènes so- ciaux qui se passent chez nous. Elle nous rappelle cette vaste oeuvre de collaboration qu'a été sur le plan économique, la collection «Notre Milieu» qui elle aussi présageait l'apparition de chercheurs parmi les nôtres.

Mais une révolution de ce genre, en appelle une autre qui, je crains, n'est pas prête à se faire. C'est l'appréciation à l'autre bout de la chaîne, au plan du public d'une oeuvre de cette envergure. Gardons au moins l'espoir qu'au niveau de ceux qui s'occupent de choses sociales, le livre soit lu et discuté. Ce cycle fermé avec succès, encouragera peutêtre à étendre à d'autres phénomènes sociaux de chez nous l'activité de nos chercheurs actuels, à en susciter d'autres.

\section{RAYMOND GÉRIN, secrétaire Centre des Patrons Chrétiens}

\section{PUBLICATIONS RECENTES}

\section{Généralités}

Research on Immigrant Adiustment and Ethric Groups, An Annual Bibliography June 1954-1955, Research Division, Canadian Citizenship Branch, Dept. of Citizenship and Immigration, Ottawa, Decєmber $1955,33 \mathrm{pp}$.

Les iournaux d'entreprise (Cours de formation dans la rédaction des journaux d'entreprise). Numéro spécial du Bulletin Social des Industriels, 71, Ave de Cortenberg, Bruxelles, Belgique, mars i 956 , pp. 570-615.

a) Le chef d'entreprise et le journal d'entrenrise, M. Vincent Levaux, pp. 571-574.

b) Le iournal d'entreprise et la législation sur les délits de presses, Me Edgard Thomas, pp. 574-580.

c) Rédaction du iournal d'entreprise, M.R. Janvier, pp. 580-582.

d) La présentation iournalistique du iournal d'entreprise, M. J. de Spot, pp. 582-588.

e) La mission sociale et psychologique du iournal d'entreprise, R.C. LiGoris, pp. 588-597.

f) La matière du iournal d'entreprise, M. Maillard, pp. 597-599. 